\title{
Effect of Linear Vestibular Stimulation on Behavioral Parameters in Young Adults
}

\author{
Sai Sailesh Kumar Goothy ${ }^{1 *}$, Archana R $^{2}$ and Mukkadan JK ${ }^{3}$ \\ ${ }^{1}$ Department of Physiology, Vishnu Dental College, Vishnupur, Bhimavaram, West \\ Godavari District, India \\ 2Department of Physiology, Saveetha Medical College, Saveetha Institute of Medical \\ and Technical Sciences, Thandalam, Chennai, India \\ ${ }^{3}$ Department of research, Little Flower Medical Research Centre, Angamaly, India
}

Research Article

Volume 4 Issue 4

Received Date: July 12, 2019

Published Date: August 16, 2019

DOI: $10.23880 / \mathrm{ijbp}-16000167$

*Corresponding author: Dr. Sai Sailesh Kumar G, Assistant Professor \& HOD (I/C), Department of Physiology, Vishnu Dental College, Vishnupur, Bhimavaram, West Godavari District, Andhra Pradesh, India, Email: dr.saisailesh@gmail.com

\section{Abstract}

Introduction: Vestibular stimulation facilitates thalamo-cortical-synchronization, decreases latency of sleep and increases duration of deep sleep stages. Vestibular stimulation regulates eating behavior through its connections with hypothalamus, dorsal raphe nucleus, nucleus tractussolitarius, locus-coeruleus and hippocampal formation. The present study was designed to evaluate the effect of vestibular stimulation on behavioral parameters in young adults.

Materials and methods: 240 (120 males and 120 females) participants satisfying both inclusion and exclusion criteria were included in the study.Vestibular stimulation was given by making the participants swing on a swing, according to their comfort. This was a longitudinal follow-up study in which, participants were assessed three times.

Results: There was significant improvement in sleep quality followed by vestibular stimulation in both male and female participants. Day time sleepiness and susceptibility to eating disorders was significantly reduced followed by vestibular stimulation in both male and female participants.

Conclusion: Vestibular stimulation may be considered as an adjunctive therapy for stress management. Further, the study could be taken up to learn the effect of vestibular stimulation in different age groups and further awaits to explore the research based evidence in learning the molecular pathways linking vestibular stimulation and its potential in stress management.

Keywords: Vestibular stimulation; Swing; Eating behavior; Sleep; Stress

\section{Introduction}

Psychological health of young adults is a neglected issue in public health care. Depression, anxiety and stress are key indicators of psychological health [1]. Sleep is a state of decreased alertness, which is regulated by circadian rhythm. Sleep is essential for our survival as we spend one-third of our life in sleep. Sleep maintains 


\section{International Journal of Biochemistry \& Physiology}

physical and mental health and contributes to wellbeing [2]. Sleep quality is a measure of subjective and objective outcomes of the sleep. Poor sleep quality is a common problem in young adults globally [3] and prevalence of sleep disorders are higher in young adults of India [4]. Sleep deprivation leads to depression and adversely affects cognition, which may result in decrease in the academic performance. Sleep deprivation in young adults may be due to the academic and examination stressor [5] non-academic diversions. Sleep deprivation may increase daytime sleepiness, which may further contribute to increased risk of cardiovascular diseases, metabolic syndrome, immunological problems and psychiatric illness [6]. Poorly managed stress has been reported to decrease the quality of sleep and sleep deprivation would be the major cause for daytime sleepiness. Excessive daytime sleepiness increases negative feelings, adversely affects cognitive functions and decrease quality of life, which may further lead to stress [7]. Stress may alter eating behavior of the individual and increases the risk of obesity [8]. Emotional eating, uncontrolled eating and increased tendency to eat non-nutritious food were observed in young adults with high stress [9]. Vestibular stimulation promotes sleep through relaxation effect, through direct and indirect connections with amygdala, sensory systems, thalamus, hypothalamus and brain stem areas [10]. Swinging has been reported to alter physiological parameters of sleep [11]. Vestibular stimulation facilitates thalamo-cortical-synchronization, decreases latency of sleep and increases duration of deep sleep stages [11]. Vestibular stimulation regulates eating behavior through its connections with hypothalamus, dorsal raphe nucleus, nucleus tractus solitarius, locuscoeruleus and hippocampal formation [12]. The present study was designed to evaluate the effect of vestibular stimulation on behavioral parameters in young adults.

\section{Materials and Methods}

\section{Participants}

A total of 300 (130 males and 170 females) young adults were screened. 240 (120 males and 120 females) participants satisfying both inclusion and exclusion criteria were included in the study. A detailed medical history was obtained from all participants and standard physical examination was conducted. Written informed consent was obtained from all the participants included in the study. Selected participants were randomly assigned to four groups by simple random sampling.

Group Con-M ( $=60)$ : Control male group (no vestibular stimulation was given)
Group Con-F ( $\mathrm{n}=60)$ : Control female group (no vestibular stimulation was given)

Group Exp-M ( $\mathrm{n}=60)$ : Experimental male group (vestibular stimulation was given)

Group Exp-F ( $\mathrm{n}=60)$ : Experimental female group (vestibular stimulation was given).

\section{Inclusion and Exclusion Criteria}

Healthy young adults in the age group of 18-24 years who were willing to participate in the study were included in the study. Individuals suffering from any somatic or mental disorders, those with ear infections or any vestibular disturbances, visual disorders, cardiorespiratory disorders were excluded from this study.

\section{Study Design}

This was a longitudinal follow-up study in which, participants were assessed three times. The first assessment was performed during regular classes (with no examination in preceding two weeks and forth coming two weeks), these are pre-intervention values. The second assessment was performed eight months after the intervention (during regular classes), and third assessment was performed sixteen months after the intervention in stressed state (A week before the University examinations).

\section{Vestibular Stimulation}

Vestibular stimulation was given by making the participants swing on a swing, according to their comfort (front and back direction) once a day, for 5 days in a week at their leisure timings (8:30-9:30 am, 11:00-12:00 am, 1:00-2:00 pm and 4:00-5:00 pm) in four groups $[13,14]$.

\section{The Pittsburgh Sleep Quality Index (PSQI)}

The Pittsburgh Sleep Quality Index (PSQI) is a standard questionnaire used to assess sleep quality and quantity for the last month. It consists of 19 self-rated questions to assess seven components of sleep (sleep quality, sleep latency, sleep duration, habitual sleep efficiency and sleep disorders, use of sleep medications, daily sleep disturbance, daily sleep duration)and 5 questions to be rated by roommate or bed partner. The self-rated questions only considered for scoring. Each component score ranges from 0 (no difficulty in sleep) to 3 (severe difficulty) and scores of the seven components has to be added to obtain the global score which ranges from 0 (better) to 21 (worse). The global score of $\leq 5$ indicates good sleep quality [15]. 


\section{International Journal of Biochemistry \& Physiology}

\section{The Epworth Sleepiness Scale}

The Epworth Sleepiness scale (ESS) is a selfadministered questionnaire that consists of eight questions to assess the daytime sleepiness. The participants were asked to respond on a four point Likert scale ranges from 0 (would never doze) to 3 (high chance of dozing). The ESS score ranges from 0-24. Higher ESS scores indicate higher daytime sleepiness $[16,17]$.

\section{The Eating attitude test (EAT-26)}

EAT-26 consists of 26 questions to screen the susceptibility to eating disorder that needs professional attention. The participants were asked to respond each question on a four point Likert scale ranges from 0 (never) to 3 (always). The scoring of question 26 was reversed and total score ranges from 0 to 78 . A score of 20 and above indicates susceptibility to eating disorder [18].

\section{Ethical considerations}

The present study was approved by the Institutional Ethics Committee (Angamaly, Kerala, India; No: IEC Jan/10/2014; dated $10^{\text {th }}$ of January 2014). Participants were recruited after taking the written informed consent. Consent form was prepared in two languages (English and Malayalam). Permission was also obtained from the management of Little Flower Hospital and Research Centre (dated: $10^{\text {th }} / J a n / 2014$ ) for conducting the study. Confidentiality of the data was maintained.

\section{Statistics}

Data was analyzed by Sigma Plot 13.0 (Systat software, USA). Median and percentile of all the observations were calculated. Friedman repeated measures analysis of variance on ranks was used to observe the significance of difference in the median values among the groups. Multiple comparisons were performed by using StudentNewman-Keuls (SNK) method. Mann-whitney rank sum test was used to observe the significance of difference between the two groups. $p<0.05$ was considered as significant

\section{Results}

\section{The Pittsburgh sleep quality index (PSQI) (score)}

The effectiveness of vestibular stimulation in young adults on the Pittsburgh sleep quality index score was presented in table 6.7. The median PSQI score in the control male group was 7. After 8 months there was no change in the PSQI score whereas after 16 months, there was increase in the PSQI score which was statistically significant $(p<0.001)$. The median PSQI score in the experimental male group was 7. After 8 months, there was a decrease in the PSQI score whereas after 16 months, there was a slight decrease in the PSQI score. The decrease in the PSQI score was statistically significant $(p<0.001)$. The median PSQI score in the control female group was 7. After 8 months there was no change in the PSQI score whereas after 16 months, there was an increase in the PSQI score which was statistically significant $(p<0.001)$.The median PSQI score in the experimental female group was 7. After 8 months, there was a decrease in the PSQI score whereas after 16 months, there was a slight decrease in the PSQI score. The decrease in the PSQI score was statistically significant $(p<0.001)$. After 8 months there was a significant decrease in the PSQI score in the experimental male ( $\mathrm{T}=5184 ; p<0.001)$ and female $(\mathrm{T}=5113 ; p<0.001$ groups when compared to control male and female groups.

\section{The Epworth Sleepiness Scale (ESS) (Score)}

The effectiveness of vestibular stimulation in young adults on the Epworth sleepiness scale score was presented in table 6.7. The median ESS score in the control male group was 8 . After 8 months, there was no change in the ESS score whereas after 16 months, there was increase in the ESS score which was statistically significant $(p<0.001)$. The median ESS score in the experimental male group was 8 . After 8 months, there was a decrease in the ESS score whereas after 16 months, there was a slight decrease in the ESS score. The decrease in the ESS score was statistically significant $(p<0.001)$. The median ESS score in the control female group was 8. After 8 months, there was no change in the ESS score whereas after 16 months, there was increase in the ESS score which was statistically significant $(p<0.001)$. The median ESS score in the experimental female group was 8. After 8 months, there was a decrease in the ESS score whereas after 16 months, there was a slight decrease in the ESS score. The decrease in the ESS score was statistically significant $(p<0.001)$. After 8 months there was a significant decrease in the PSQI score in the experimental male $(\mathrm{T}=4894 ; p<0.001)$ and female ( $\mathrm{T}=4890 ; p<0.001$ groups when compared to control male and female groups.

\section{The Eating attitude test (EAT-26) (score)}

The effectiveness of vestibular stimulation in young adults on the Eating attitude test score was presented in 


\section{International Journal of Biochemistry \& Physiology}

table 6.7. The median EAT-26 score in the control male group was 13.000. After 8 months, there was a decrease in the EAT-26 score which was statistically significant $(p<0.001)$. After 16 months, there was an increase in the EAT-26 score which was statistically significant $(p<0.001)$. The median EAT-26 score in the experimental male group was 13 . After 8 months, there was a decrease in the EAT-26 score whereas after 16 months, there was a slight decrease in the EAT-26 score. The decrease in the EAT-26 score was statistically significant $(p<0.001)$. The median EAT-26 score in the control male group was 15. After 8 months, there was a slight decrease in the EAT-26 score which was not statistically significant. After 16 months, there was an increase in the EAT-26 score which was statistically significant $(p<0.001)$. The median EAT26 score in the experimental female group was 15 . After 8 months, there was a decrease in the EAT-26 score whereas after 16 months, there was a slight decrease in the EAT-26 score. The decrease in the EAT-26 score is statistically significant $(p<0.001)$. After 8 months there was a significant decrease in the EAT-26 score in the experimental male $(\mathrm{T}=4432 ; p<0.001)$ and female ( $\mathrm{T}=4896 ; p<0.001$ groups when compared to control male and female groups.

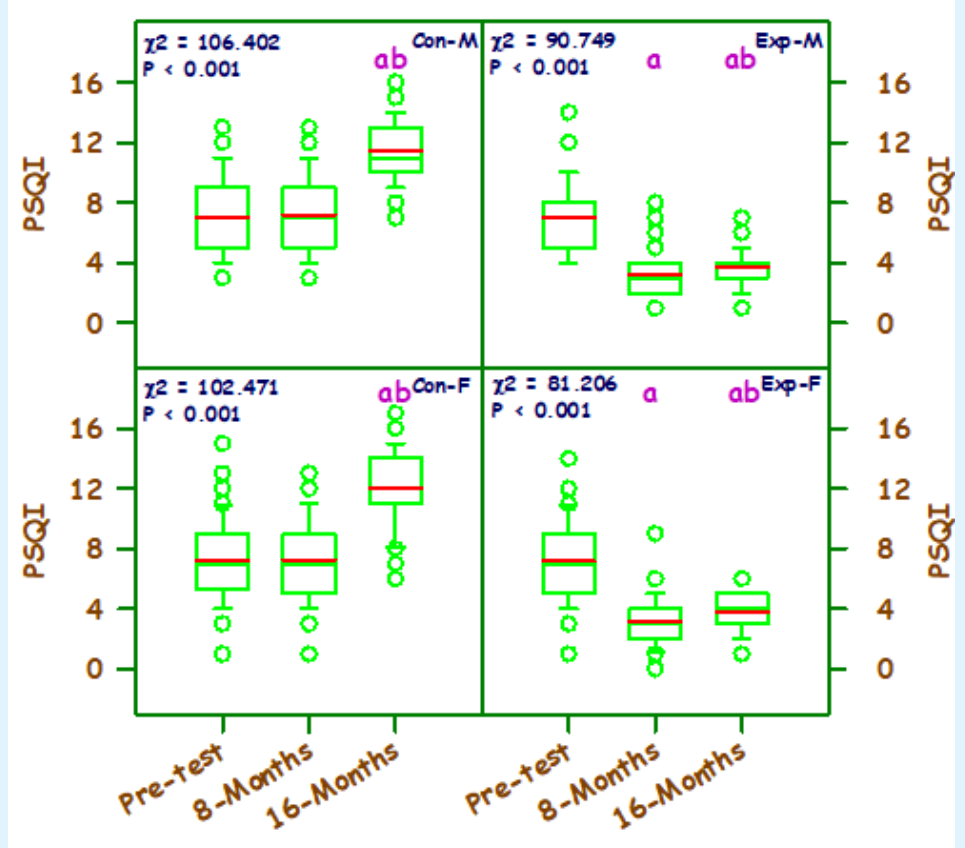

Figure 1: Effectiveness of vestibular stimulation in young adults on the Pittsburgh sleep quality index (PSQI) (score). Con = Control; Exp = Experimental; $\mathrm{M}=$ Male; $\mathrm{F}=$ Female

The middle green line is the median and the red line is the mean.

$\mathrm{n}$ - Control $=60$ each; Experimental $=60$ each

The ' $\chi 2$ ' and ' $P$ ' values are by Friedman RM ANOVA on ranks with SNK multiple comparison test.

aSignificantly different from the pre-test group.

bSignificantly different from the 8 months group. 


\section{International Journal of Biochemistry \& Physiology}

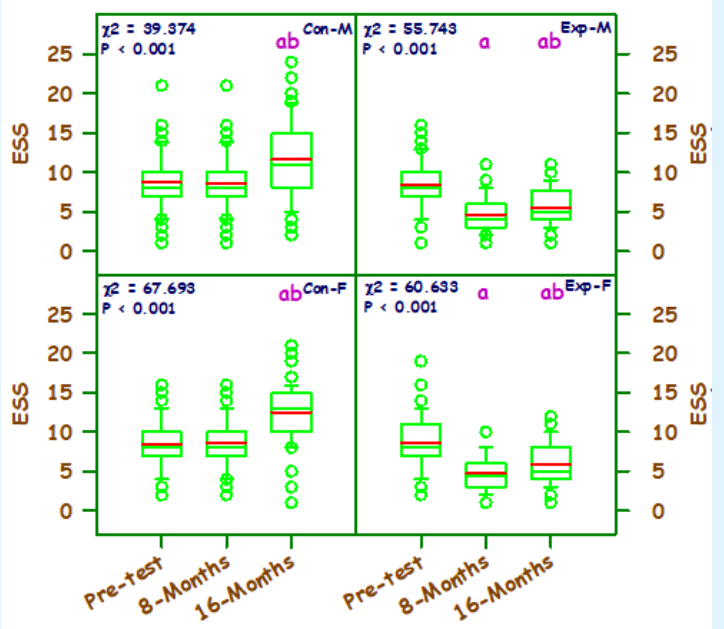

Figure 2: Effectiveness of vestibular stimulation in young adults on the Epworth sleepiness scale (ESS) (score). Con = Control; Exp = Experimental; $\mathrm{M}=$ Male; $\mathrm{F}=$ Female

The middle green line is the median and the red line is the mean.

$\mathrm{n}$ - Control $=60$ each; Experimental $=60$ each

The ' $\chi 2$ ' and 'P' values are by Friedman RM ANOVA on ranks with SNK multiple comparison test.

aSignificantly different from the pre-test group.

bSignificantly different from the 8 months group.

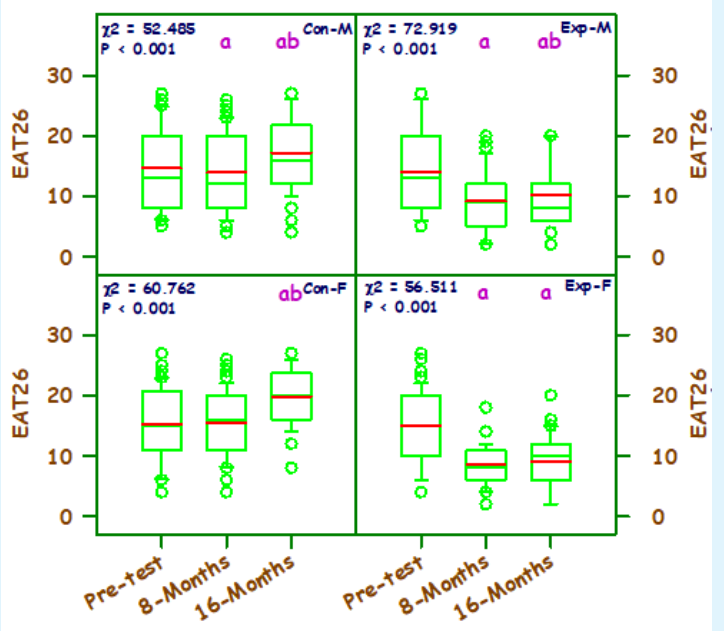

Figure 3: Effectiveness of vestibular stimulation in young adults on Eating attitude test (EAT-26) (score).

Con = Control; Exp = Experimental; $\mathrm{M}=$ Male; $\mathrm{F}=$ Female

The middle green line is the median and the red line is the mean.

$\mathrm{n}$ - Control $=60$ each; Experimental $=60$ each

The ' $\chi 2$ ' and ' $\mathrm{P}$ ' values are by Friedman RM ANOVA on ranks with SNK multiple comparison test.

aSignificantly different from the pre-test group.

bSignificantly different from the 8 months group. 


\section{International Journal of Biochemistry \& Physiology}

\section{Discussion}

Serotonin, which is reported to cause changes in the behavior and poor self-regulation, is known to play a key role in modulation of sleep and personality [19]. Though pharmacological preparations are available to improve the quality of sleep, their long-term use has limited efficacy and several undesired effects. Hence, nonpharmacological and effective interventions are necessary to improve the life style by enhancing the quality of sleep. In the present study, vestibular stimulation was tested as one such effective intervention to improve direct and associated index of sleep. Consistent with previous observations in this study, overall sleep quality was significantly improved and day time sleepiness was significantly decreased following vestibular stimulation in male and female intervention groups. Although linear swinging and rocking chairs are well known to induce sleep [20], the pathways involved are not clearly known [10]. The gross observations on the beneficial effects of vestibular stimulation on various sleep parameters are consistent with established research reporting sleep promoting effects of vestibular stimulation by modulation of the activity of different brain areas related to sleep and wakefulness such as supra chiasmatic nucleus, dorsal raphe nucleus, intergeniculate leaflet, nucleus of tractus solitaries, posterior hypothalamus, hippocampal formation and pontine reticular formation [21]. These beneficial effects are also mediated by release of serotonin, which is further converted into melatonin in the pineal gland and eventually facilitates sleep [22]. Nucleus of tractus solitaries, hippocampal neurons and hypothalamus may also be modulated by vestibular stimulation in improving sleep parameters [23]. In addition to these mechanisms, vestibular system also directly promotes sleep through inducing relaxation [10].

Relaxation is known to induce sleep through reducing the physical tension and limiting the thought process [24]. Consistent with this study, rotatory vestibular stimulation and linear acceleration was reported to increase the release of acetylcholine in Wister albino rats [25] and facilitate the REM sleep [26]. Similar to vestibular stimulation, bilateral electrical stimulation of the vestibular apparatus, rocking bed therapy and sinusoidal vestibular stimulation also is reported to decrease sleep latency [27]. The current study results are further in accordance with earlier studies reporting overall improvement in the sleep quality and decrease in daytime sleepiness following vestibular stimulation.
This study also evaluated EAT-26 score as an indirect consequence of altered sleep pattern and tested the effectiveness of vestibular stimulation in improving EAT26 score. Vestibular stimulation is reported to modulate feeding behavior by modulating the activity of arcuate nucleus, dorsal raphe nucleus and NTS [28]. Stimulation of vestibular apparatus also promotes good feeding habits through relieving stress and promoting sleep [29]. Arcuate nucleus is the key area of hypothalamus, which regulates the set-point [30]. Vestibular system sends projections to the brainstem areas, which are involved in homeostasis [31] and vestibular stimulation is reported to increase serotonin levels and activate arcuate nucleus [28]. Vestibular stimulation activates the vagal nerve, leading to increase in secretion of cholecystokinin and ghrelin and decrease in leptin levels and plays a key role in satiety signal and energy balance [32]. Vagal stimulation also increases release of insulin, which acts on hypothalamus and decreases food intake [33]. Vestibular stimulation regulates HPA axis and decreases the cortisol levels with in normal limits, which is consistent with observations in this study. In contrast dysregulation of HPA axis was associated with obesity of upper body with increase in the cortisol levels [34]. Consistent with the observations in this study, longitudinal caloric vestibular stimulation was reported to significantly decrease the total cholesterol, triglycerides, high and low density lipoproteins levels in albino rats [35]. Unilateral electrical stimulation of vestibular nuclei following ingestion of saccharin was reported to induce taste eversion to this substance [36]. While repeated electrical stimulation of vestibular nerve decreased body fat in humans [12]. This study supports the long-term practice of vestibular stimulation by young adults to increase in the sleep quality, decrease in the daytime sleepiness and susceptibility to eating disorder. Vestibular stimulation is a simple, cheap, and easy to follow and comfortably practice to combat stress associated disturbance in sleep patterns and its associated events. In summary, this study provides supporting evidence for vestibular stimulation as an efficient method to enhance memory and reaction time and to effectively prevent stress-induced changes on memory and reaction time, sleep and eating behavior. Hence vestibular stimulation is recommended as a simple approach to improve the memory, reaction time and academic performance, which could consequently improve the quality of life.

\section{Conclusion}

Vestibular stimulation may be considered as an adjunctive therapy for stress management. Further, the 


\section{International Journal of Biochemistry \& Physiology}

study could be taken up to learn the effect of vestibular stimulation in different age groups and further awaits to explore the research based evidence in learning the molecular pathways linking vestibular stimulation and its potential in stress management.

\section{References}

1. Teh CK, Ngo CW, Zulkifli RAB, Vellasamy R, Suresh K (2015) Depression, Anxiety and Stress among Undergraduate Students: A Cross Sectional Study. Open J Epidemiol 5(4): 260-268.

2. Miller MA (2015) The Role of Sleep and Sleep Disorders in the Development, Diagnosis, and Management of Neurocognitive Disorders. Front Neurol 6: 224.

3. Abdulghani HM, Alrowais NA, Bin-Saad NS, Al-Subaie NM, Haji AMA, et al. (2012) Sleep disorder among medical students: relationship to their academic performance. Med Teach 34: 37-41.

4. Kaur G, Singh A (2017) Excessive daytime sleepiness and its pattern among Indian college students. Sleep Med 29: 23-28.

5. Giese M, Unternaehrer E, Brand S, Calabrese P, Holsboer-Trachsler E, et al. (2013) The Interplay of Stress and Sleep Impacts BDNF Level. PLOS ONE $8(10)$.

6. Giri P, Baviskar M, Phalke D (2013) Study of Sleep Habits and Sleep Problems Among Medical Students of Pravara Institute of Medical Sciences Loni, Western Maharashtra, India. Ann Med Health Sci Res 3(1): 5154.

7. Roth $\mathrm{T}$ (2015) Effects of Excessive Daytime Sleepiness and Fatigue on Overall Health and Cognitive Function. J Clin Psychiatry 76(9).

8. Deliens T, Clarys P, De Bourdeaudhuij ID, Deforche B (2014) Determinants of eating behaviour in university students: a qualitative study using focus group discussions. BMC Public Health 14: 53.

9. Penaforte FR, Matta NC, Japur CC (2016) Associacao entre estresse e comportamento alimentarem estudantes universitarios. Demetra Aliment Nutr \& Saud 11(1).

10. Omlin X, Crivelli F, Heinicke L, Zaunseder S, Achermann P, et al. (2016) Effect of Rocking Movements on Respiration. PLOS ONE 11(3).
11. Bayer L, Constantinescu I, Perrig S, Vidal PP, Muhlethaler M, et al. (2011) Rocking synchronizes brain waves during a short nap. Curr Bio 21(12): 461462.

12. McGeoch PD, McKeown J, Peterson H, Ramachandran VS (2017) Modulation of Body Mass Composition using Vestibular Nerve Stimulation. bioRxiv.

13. Sailesh KS, Mukkadan JK (2015) Controlled Vestibular Stimulation, Standardization of A Physiological Method to Release Stress in College Students. Indian J Physiol Pharmacol 59(4): 436-441.

14. Sailesh KS (2014) Controlled Vestibular Stimulation: A Physiological Method of Stress Relief. J Clin Diagn Res 8(12): BM01-BM02.

15. Buysse DJ, Reynolds CF, Monk TH, Berman SR, Kupfer DJ (1989) The Pittsburgh Sleep Quality Index: a new instrument for psychiatric practice and research. Psychiatry Res 28(2): 193-213.

16. Johns MW (1991) A new method for measuring daytime sleepiness: The Epworth sleepiness scale. Sleep 14(6): 540-545.

17. Johns MW (2000) A sleep physiologist's view of the drowsy driver. Transp Res Part F 3(4): 241-249.

18. Garner DM, Olmsted MP, Bohr Y, Garfinkel PE (1982) The eating attitudes test: psychometric features and clinical correlates. Psychol Med 12(4): 871-878.

19. Carver CS, Johnson SL, Joormann J (2009) Two-Mode Models of Self-Regulation as a Tool for Conceptualizing Effects of the Serotonin System in Normal Behavior and Diverse Disorders. Curr Dir Psychol Sci 18(4): 195-199.

20. Bayer L, Constantinescu I, Perrig S, Vienne J, Vidal PP, et al. (2011) Rocking synchronizes brain waves during a short nap. Curr Biol 21(12): 461-462.

21. Horowitz SS, Blanchard JH, Morin LP (2004) Intergeniculate leaflet and ventral lateral geniculate nucleus afferent connections: An anatomical substrate for functional input from the vestibulovisuomotor system. J Comp Neurol 474(2): 227-245.

22. Zhdanova IV, Lynch HJ, Wurtman RJ (1997) Melatonin: a sleep-promoting hormone. Sleep 20(10): 899-907. 


\section{International Journal of Biochemistry \& Physiology}

23. Saman Y, Bamiou DE, Gleeson M, Dutia MB (2012) Interactions between Stress and Vestibular Compensation-A Review. Front Neurol 3: 116.

24. Dusek JA, Benson H (2009) Mind-Body Medicine. Minn Med 92: 47-50.

25. Devi Np, Mukkadan JK (2016) Impact of rotatory vestibular stimulation and curcuma longa on spatial learning and memory in wistar albino rats. Asian J Pharm Clin Res 9(2): 167-173.

26. Vazquez J, Baghdoyan HA (2001) Basal forebrain acetylcholine release during REM sleep is significantly greater than during waking. Am J Physiol Regul Integr Comp Physiol 280(2): 598-601.

27. Krystal AD, Zammit GK, Wyatt JK, Quan SF, Edinger JD, et al. (2010) The effect of vestibular stimulation in a four-hour sleep phase advance model of transient insomnia. J Clin Sleep Med 6(4): 315-321.

28. Fuller PM, Jones TA, Jones SM, Fuller CA (2004) Evidence for macular gravity receptor modulation of hypothalamic, limbic and autonomic nuclei. Neuroscience 129(2): 461-471.

29. Tamashiro KL, Sakai RR, Shively CA, Karatsoreos IN, Reagan LP (2011) chronic stress, metabolism, and metabolic syndrome. Stress Amst Neth 14(5): 468474 .

30. Wang D, He X, Zhao Z, Feng Q, Lin R, et al. (2015) Whole-brain mapping of the direct inputs and axonal projections of POMC and AgRP neurons. Front Neuroanat.

31. Balaban CD, Yates BJ (2004) Vestibuloautonomic Interactions: A Teleologic Perspective in the vestibular system springer handbook of auditory research. 19: 286-342.

32. Bugajski AJ, Gil K, Ziomber A, Zurowski D, Zaraska W, et al. (2007) Effect of long-term vagal stimulation on food intake and body weight during diet induced obesity in rats. J Physiol Pharmacol 58(1): 5-12.

33. Filippi BM, Bassiri A, Abraham MA, Duca FA, Yue JT, et al. (2014) Insulin signals through the dorsal vagal complex to regulate energy balance. Diabetes 63(3): 892-899.

34. Bose M, Olivan B, Laferrere B (2009) Stress and obesity: the role of the hypothalamic-pituitaryadrenal axis in metabolic disease. Curr Opin Endocrinol Diabetes Obes 16(5): 340-346.

35. Sadanandan NN, Archana R, Sailesh KS, Mukkandan JK, Antont NJ (2015) Antihyperlipidemic effect of vestibular stimulation in wistar albino rats. Int J Res Ayurveda Pharm 6: 509-512.

36. Brozek G (1982) Electrophysiological analysis of conditioned taste aversion in rats. Acta Neurobiol Exp 42(1): 29-41. 